Interfaces

Gestes de surface : Touching Reality de Thomas Hirschhorn et What Shall We Do Next? de Julien Prévieux

\title{
Pascale Borrel
}

\section{(2) OpenEdition}

\section{Journals}

Édition électronique

URL : http://journals.openedition.org/interfaces/601

DOI : 10.4000/interfaces.601

ISSN : 2647-6754

Éditeur :

Université de Bourgogne, Université de Paris, College of the Holy Cross

\section{Édition imprimée}

Date de publication : 21 décembre 2018

Pagination : 55-65

ISSN : 1164-6225

\section{Référence électronique}

Pascale Borrel, « Gestes de surface : Touching Reality de Thomas Hirschhorn et What Shall We Do Next? de Julien Prévieux », Interfaces [En ligne], 40 | 2018, mis en ligne le 21 décembre 2018, consulté le 06 janvier 2021. URL : http://journals.openedition.org/interfaces/601 ; DOI : https://doi.org/10.4000/ interfaces.601

\section{(c) $(0)$}

Les contenus de la revue Interfaces sont mis à disposition selon les termes de la Licence Creative Commons Attribution 4.0 International. 


\title{
GESTES DE SURFACE : TOUCHING REALITY DE THOMAS HIRSCHHORN ET WHAT SHALL WE DO NEXT? DE JULIEN PRÉVIEUX
}

\author{
Pascale Borrel \\ Université Rennes 2
}

\begin{abstract}
Résumé: Si l'on peut mettre en relation des œuvres aussi différentes que Touching Reality de Thomas Hirschhorn (2012) et What Shall We Do Next de Julien Prévieux (2006-2014) c'est parce qu'elles consistent, l'une et l'autre, en une représentation de gestes définis par les technologies numériques. Touching Reality est une vidéo qui, en une projection de taille imposante, montre des photographies de corps mutilés qu'une main de femme fait défiler et agrandit sur un écran tactile. What Shall We Do Next comprend un film d'animation et une pièce chorégraphique consistant en une suite de mouvements « à vide »; il s'agit de gestes que les fabricants d'ordinateurs et de consoles de jeux ont fait breveter auprès de l'agence américaine USPO, des manipulations relatives à des appareils pour l'heure à l'état d'étude. C'est de la représentation que ces artistes ont choisi de donner de ces nouvelles « techniques du corps » qu'il sera question: du type de mouvements que ces œuvres mettent en évidence et de la manière dont ces artistes articulent ces manipulations avec le réel. L'action du corps se réduit ici à celle de la main. Thomas Hirschhorn présente et Julien Prévieux suggère son glissement sur les écrans; ils montrent différentes orchestrations des doigts sur leur surface lisse et font percevoir la singularité de ces manipulations familières ou à venir, singularité qui réside dans une combinaison de précision délicate et de systématisme froid. En associant à ces effleurements un défilement d'images de corps détruits trouvés sur les réseaux sociaux, Thomas Hirschhorn indique sur un mode délibérément cru la violence qui réside dans cette « atopie fugitive» (Mondzain). Si l'œuvre de Julien Prévieux consiste à ne pas figurer explicitement ce réel, c'est toutefois à une forme équivalente de violence que cet enchainement de gestes atones semble se référer.
\end{abstract}

Abstract: Both Touching Reality by Thomas Hirschhorn (2012) and What Shall We Do Next? by Julien Prévieux (2006-2014) present gestures determined by digital technology. Touching Reality is a video which shows large-scale images of mutilated bodies that a woman's hand enlarges on a tactile screen. What Shall We Do Next? consists in an animated short film and a choreographic creation based on movements repeated in empty space. They are gestures that computer and video games programmers have had patented with the American agency USPO, gestures relating to devices that are for the moment still being developed. Our aim is to study how these artists have chosen to represent these new body gestures and the way they impact on the real world around us. The focus is here on the hand. Thomas Hirschhorn presents the gesture of the moving hand on the screen, and Julien Prévieux suggests how it slides on the screen. They show how the movements of the fingers on the smooth surface, whether already familiar or still to come, are singular, because they 
are both delicately precise, but also systematically cold. By associating these light touches to the photos of destroyed bodies, found on social networks, Thomas Hirschhorn reveals the inherent violence which resides in this "fugitive atopia" (Mondzain). Though Julien Prévieux does not crudely represent it, he seems to refer to the same kind of underlying violence.

Touching Reality ${ }^{1}$ de Thomas Hirschhorn est une vidéo qui, en une projection de taille imposante, montre la surface d'un écran tactile sur laquelle une main de femme fait défiler et agrandit des images de corps détruits. What Shall We Do Next? de Julien Prévieux est le titre commun à trois œuvres $^{2}$ - un film d'animation, une pièce chorégraphique filmée et des performances dansées (dont il ne sera pas question ici) - conçues à partir de mouvements que les concepteurs d'appareils numériques font breveter. Ces œuvres, si différentes dans leurs formes, représentent donc les manipulations que requièrent les claviers, les écrans tactiles ou encore les joysticks. Parler de " geste », dans ce contexte, c'est départir ce mot de l'expressivité et de l'amplitude qu'il laisse entendre puisque ces mouvements-là sont produits et contraints par des appareils, puisqu'ils sont programmés au sens propre du terme, « écrits à l'avance », puisqu'ils relèvent de l'automatisme tel que Gilbert Simondon en parle : « L'homme, interprète des machines, est aussi celui qui, à partir de ses schèmes, a fondé des formes rigides qui permettent à la machine de fonctionner. La machine est un geste humain déposé, fixé, devenu stéréotypie et pouvoir de recommencement» (138).

Les machines auxquelles Hirschhorn et Prévieux se réfèrent, impliquent un certain type de gestes dont la particularité est perceptible dans les sens du mot « digital» en français. Dans son sens initial, il désigne « ce qui se rapporte aux doigts » et il est également employé comme synonyme de « numérique ». Dans une volonté de clarté, l'Académie française recommande de renoncer à l'emploi de « digital » dans le cadre des technologies dites « numériques ». Toutefois, on peut considérer que l'utilisation conjointe des deux mots, dans ce contexte technique-là, est l'expression de niveaux distincts : « Numérique tend

Touching Reality de Thomas Hirschhorn (2012) est une vidéo couleur sans son, en boucle (4'45'). URL : https:// vimeo.com/55482318 (page consultée le 14 novembre 2018).

What Shall We Do Next $\neq \neq 1$ de Julien Prévieux est une animation 3D noir et blanc, en cours depuis 2006. Vidéo sans son, projetée sur écran LCD transparent et rétroprojecteur (3'54'’). URL : https://vimeo.com/59793317.

What Shall We Do Next? $\neq 2$ (2014) est une vidéo HD/2K, en couleur (16'47')). URL : https://vimeo.com/111013619 (page consultée le 14 novembre 2018).

What Shall We Do Next? $\neq 3$ est une performance constituée de 3 modules de 7 minutes environ chacun; le premier est fait de gestes issus de films de fiction hollywoodiens (de 1950 à aujourd'hui) ; le second est la reprise d'une œuvre de Martha Graham (en référence aux questions juridiques relatives à la propriété des gestes qui se sont posées à la mort de la chorégraphe); le troisième est composé des derniers gestes que Prévieux a extraits du site USPTO. 
à renvoyer de fait au technologique, à la dimension discrète de la technologie, celle que manipulent les ingénieurs et qui reste intangible. Digital semblerait concerner plutôt l'usager dans son expérience de cette technologie numérique. [...] L'imaginaire linguistique du digital est lié à la dimension expériencielle et à l'usage alors que l'imaginaire linguistique du numérique est lié à l'industrie et à l'encodage » (Ropars). Le mot « digital» fait percevoir l'importance que les appareils donnent au toucher ; il évoque les calculs complexes dont ces gestes légers, ces effleurements dépendent. Chacune à leur manière, les œuvres de Hirschhorn et de Prévieux mettent en évidence le caractère économe et subtil de cette gestuelle, ils en expriment la délicatesse ou l'élégance. Il s'agira de mettre en évidence les moyens que les artistes emploient pour souligner ces qualités et surtout les réalités auxquelles ils les associent. Si Touching Reality s'oppose, par ses images crues, à l'univers aseptisé de What Shall We Do Next?, il semble cependant que ces œuvres manifestent, l'une et l'autre, les violences sourdes qui existent sous la douceur de ces gestes de surface.

\section{Caresses}

Touching Reality montre un mouvement désormais familier, celui qui permet de faire défiler et d'agrandir des images sur une tablette ou un Smartphone. Le déplacement de l'index de droite à gauche transpose, sur la surface lisse de l'appareil, la manipulation du volume d'un livre. Mais la pince qui s'ouvre et se ferme dans le « pinch \& expand » (la fonction « pincer et agrandir ») rompt l'analogie entre l'écran et l'objet de papier, par le changement d'échelle que ce geste a la capacité de produire. L'œuvre souligne la douceur de ces manipulations qui ne rencontrent aucune espèce de résistance physique. Cette chorégraphie fluide sur la surface réactive de l'appareil est toutefois un processus ambivalent où se combinent la sensualité de l'effleurement et la raideur du systématisme et de la répétition.

Le premier volet de l'œuvre de Prévieux, What Shall We Do Next? $\neq 1$, fait percevoir ce même mélange de douceur et de rigueur. Il s'agit d'un dessin d'animation où deux mains vues en surplomb effectuent des actions plus ou moins identifiables car les appareils qu'elles sont censées faire fonctionner ne sont pas représentés. Ces manipulations, l'artiste en a trouvé la description sur le site de l'agence américaine USPTO auprès de laquelle les fabricants déposent des brevets de gestes, plusieurs années avant que les appareils soient concrétisés (quand ils le sont). Cette pratique, qui atteste de la concurrence acharnée que se livrent les firmes (on se souvient du procès qui a opposé Apple et Samsung au sujet du « glisser pour déverrouiller $»^{3}$ ) produit un répertoire de gestes potentiels,

En 2016, après plusieurs années de bataille judiciaire, Samsung gagne en appel son procès contre Apple qui avait attaqué la firme coréenne au sujet de trois brevets : une méthode de correction automatique du texte, un système d'identification d'un numéro apparaissant dans un message et, en matière de geste, le « glisser pour déverrouiller ». 
« en gestation », un « réservoir de gestes futurs, de gestes à venir » (Prévieux, entretien avec Olivier Poncet). Le travail de Prévieux consiste donc à puiser dans ce « réservoir », à utiliser des éléments élaborés par d'autres selon la logique du readymade ou plus précisément, comme le dit l'artiste, du « found gesture », au sens que Simone Forti et Yvonne Rainer ont donné à cette expression dans le contexte de la danse (entretien avec Olivier Poncet). Le dessin d'animation entretient une relation étroite avec les documents graphiques et textuels que Prévieux a rassemblés. La manière dont les mains sont figurées évoque l'économie et le schématisme qui y sont utilisés. La représentation des mouvements est déduite des descriptions que ces brevets présentent, descriptions dont l'artiste souligne la précision et la part de flou conjointes : il s'agit en effet pour l'inventeur de faire apparaître la singularité, l'originalité du geste qu'il prétend breveter tout en laissant percevoir la marge de manœuvre qu'il offre. Les deux mains animées enchaînent donc une suite de mouvements " à vide », qui permettent d'identifier la nature ou, au moins, la forme générale des objets auxquels ils se rapportent. Certains de ces mouvements renvoient à des usages familiers alors que d'autres sont des éléments de vocabulaire inconnus, au devenir incertain. À partir de gestes tributaires d'appareils différents, l'œuvre met en place un flux continu ${ }^{4}$ : les mouvements se succèdent sans heurts, sans interruption, selon un rythme constant. Ils sont dotés d'une sorte de régularité et de grâce ; ils donnent la sensation d'appartenir à un monde qui n'est pas soumis aux lois physiques et aux contingences humaines, un monde où un seul appareil, omnipotent, aurait la capacité de susciter tous ces gestes et d'accomplir leurs mystérieuses fonctions.

\section{Le futur, le passé, le présent}

Dans What Shall We Do Next? $\neq 2$, Prévieux a soumis les gestes collectés à l'épreuve de l'espace physique réel en les faisant interpréter par des danseurs. En tant que chorégraphie filmée, l'œuvre repose à la fois sur une organisation des corps en mouvement dans l'espace et sur un travail de prise de vue et de montage. Six danseurs évoluent dans une pièce assez vaste ; sur ses murs blancs ont été peints des carrés et des rectangles gris, bleus et verts ; le sol gris est marqué d'un réseau de lignes blanches qui le subdivise en grandes aires carrées. La structure géométrique de l'espace annonce la rigueur qui régit la chorégraphie, le type de motricité qui s'y manifeste. Les danseurs suivent des trajectoires rectilignes avec une certaine lenteur, droits, raides, le visage inexpressif, sans véritablement interagir les uns avec

La durée de ce flux est, par principe, toujours extensible puisque Prévieux considère l'œuvre comme une archive à compléter au fil des nouvelles publications sur USPTO. 
les autres ${ }^{5}$ et ils s'immobilisent en des poses hiératiques pour exécuter ces « gestes trouvés ». L'atonie expressive ambiante met en évidence leur diversité, leurs subtilités, d'autant que ces gestes peuvent se réaliser selon différentes configurations spatiales : du bout des doigts, avec la paume de la main, les danseurs font exister des surfaces de travail verticales et horizontales ; et les micro-gestes qu'ils dispersent dans l'espace évoquent différents modes de portabilité des appareils.

What Shall We Do Next? $\neq 2$ donne au texte un rôle important ; il est énoncé par les danseurs et par une voix off. Au commencement du film, les premiers égrènent d'une voix monocorde des nombres 2009, 2009, 2007, 2008, 2009, 2009 ... que 1'on peut supposer correspondre aux années de dépôts des brevets. Les propos que le film fait entendre par la suite se rapportent à des inventions, des prototypes ou des expériences appartenant à une sphère bien plus large que le seul cadre de ces brevets. Si Prévieux a composé une partie de ces textes à partir des discours qui font la promotion de ces appareils, il a également introduit des propos relatifs à des inventions plus anciennes comme par exemple les premiers développements de l'ordinateur Xerox Star commercialisé en 1981, le Pocket Printing Machine conçu au milieu du XIX ${ }^{\text {ème }}$ siècle, le thérémine, ce synthétiseur électronique contrôlé par des gestes, inventé en 1919 par Lev Sergueïevitch Termen... Appareils de l'avenir ou machines obsolètes, ces créations disparates ne sont pas présentées selon l'ordre chronologique de leur conception. Le régime narratif de la voix off contribue, lui aussi, à une perte des repères temporels : l'emploi du passé et du conditionnel donne la sensation que tout ce dont il est question appartient à un temps révolu, que tous ces gestes et ces usages sont vus de manière rétrospective, depuis un temps qui est pour nous le futur. Ce mode narratif, qui emprunte au récit de science-fiction, participe du projet de Prévieux consistant à « scénariser doucement» le matériau utilisé (Prévieux, entretien avec Olivier Poncet). L'objectif n'est pas de construire une histoire mais de faire émerger différents thèmes ou lieux communs de l'imaginaire technique : la recherche d'une relation immédiate, «naturelle » avec les appareils ${ }^{6}$, la déshumanisation à laquelle ceux-ci pourraient conduire ${ }^{7}$, le contrôle qu'ils permettraient d'exercer sur une humanité décérébrée... Et What Shall We Do Next? $\neq 2$ établit des analogies entre des modes opératoires du futur et des « techniques du corps » d'un tout autre registre : la solennité avec laquelle les danseurs miment des commandes à distance évoque des pratiques magiques; et la démonstration de certaines

Parfois des scènes de groupe se constituent : les danseurs miment la réalisation d'une photo de groupe ou ils rient à l'anecdote racontée par l'un deux ; ces situations de communication sont dénuées de tout effet de spontanéité et elles sont exécutées sur un mode parfaitement mécanique.

6 Par exemple : une danseuse parle du remplacement du clavier et de la souris par « des formes d'interaction plus naturelles » et les danseurs disent, de concert, « Natural User Interface ».

L'une des premières phrases du film est : «Est-ce que je salue plus souvent mes appareils numériques que mes amis?». 
inventions farfelues ${ }^{8}$ fait percevoir, dans le discours du scientifique, celui du bonimenteur. Le travail avec ces gestes brevetés ne rend pas compte seulement d'une actualité technique et économique mais aussi des idées, des fantasmes qu'une longue cohabitation avec les machines a produits.

Dans l'œuvre de Hirschhorn, en revanche, le geste numérique consiste à établir une relation immédiate, visible avec le réel, comme son titre l'indique de manière explicite. Touching Reality est la représentation d'une action qui fait apparaître sur un écran les images d'une actualité violente. L'artiste dit avoir été intéressé par ce geste " nouveau », auquel il était, en 2012, " peu habitué » (Hirschhorn, « Insoutenables destruction de corps »), ce geste qui permet de faire défiler et d'examiner des données éparses, des images en circulation.

\section{Distances}

Dans l'ensemble de son travail, Hirschhorn utilise des représentations de la violence diffusées par les médias. Il fait souvent coexister des images de presse et des images publicitaires, les violences subies que montrent les premières et les obscénités qui émanent des secondes. Dans Touching Reality, les images utilisées proviennent des réseaux sociaux, c'est-à-dire non pas d'un « régime de diffusion fortement centralisé (donc aisément contrôlable) » mais « de la logique d'éclatement et de décentralisation qui est celle d'Internet - rendant moins "tenable" le destin des objets qui y circulent » (Citton, Renverser l'insoutenable 42).

Dans un texte intitulé « Pourquoi est-il important - aujourd'hui - de montrer et regarder des images de corps humains détruits ?», Hirschhorn explique les raisons de son choix, c'est-à-dire la valeur qu'il donne à ces images. Elles ont été « faites par des non-photographes [...] par des témoins, des passants, des soldats, des agents de sécurité ou de police, des sauveteurs ou des secouristes » et leur provenance «n'est pas très claire, souvent invérifiable, et la source manque, selon notre compréhension de ce qu'est une "source" » (62). Ces images dérogent aux usages du photojournalisme car il leur manque une légende, un paratexte indiquant de quel point de vue et à quelle fin elles ont été faites. Selon Hirschhorn, leur existence trouble et marginale est également due à la violence qu'elles exhibent : le peu de visibilité que leur donne la presse, afin de ne pas « heurter la sensibilité du public ou satisfaire le voyeurisme », participe d' " une stratégie de soutien à, ou tout au moins, de non-dissuasion de l'effort de guerre » (62), stratégie qui s'est imposée en Occident depuis le 11 septembre 2001. Hirschhorn souligne donc la relation que son œuvre entretient avec un contexte politique qui 
détermine un certain usage des images. Les moyens employés, simples, directs donnent à l'œuvre une efficacité brutale : les images problématiques que l'artiste a rassemblées sont présentées dans leur « milieu », un appareil connecté, qui est aussi le cadre des pratiques quotidiennes les plus diverses. La relation entre une réalité tragique et un espace familier établie par Touching Reality peut évoquer celle que Martha Rosler a produite dans Bringing the War Home: House Beautiful, une série réalisée entre 1967 et 1972 puis remise en œuvre entre 2004 et 2008. Le principe consiste à introduire dans des vues d'espaces domestiques confortables (House Beautiful est le nom d'un magazine de décoration intérieure) des images de la guerre du Viêt-Nam et d'Irak. L'artiste les a logées dans l'encadrement des fenêtres et des éléments de décoration murale ou y a prélevé des personnages qu'elle a implantés dans ces intérieurs. Dans la première partie de Bringing the War Home, elle s'est servie de photographies parues dans la presse. Dans la seconde, le matériau employé se fait l'écho des pratiques actuelles de production et de diffusion des images ; l'artiste a associé aux espaces domestiques des publicités pour les téléphones portables et des photographies des tortures d'Abu Graib.

Bringing the War Home et Touching Reality reflètent la proximité avec différentes formes de violence que, depuis les années cinquante, les médias ont instaurées. Toutefois ces œuvres se réfèrent à ce processus de manière très différente. Rosler associe des représentations contextualisées (les guerres menées par les États-Unis) aux espaces de l'American way of life. En manifestant explicitement leur dimension critique, ces photomontages cherchent à susciter un regard distancé plutôt qu'une sensation, une émotion. Hirschhorn, en revanche, prend le parti d'une exhibition frontale, choquante. L'écran tactile a certes un rôle analogue à celui des intérieurs de Rosler, en tant que signe du quotidien ; mais plutôt que de produire une distanciation, il implique le spectateur. Cette main dont l'œuvre fait éprouver l'action dans la durée est potentiellement la nôtre. D'ailleurs, Hirschhorn indique clairement que c'est cette relation directe qu'il a voulue établir :

Je ne veux pas me distancer, je ne veux pas regarder ailleurs, je ne veux pas détourner le regard. Parfois j'entends des visiteurs dire, en regardant des images de corps humains détruits : « Je ne peux pas regarder ça, il ne faut pas que je voie ça, je suis trop sensible ». Cela permet de conserver une distance confortable, narcissique et exclusive avec la réalité d'aujourd'hui et le monde. (« Pourquoi est-il important » 66)

Ce que l'artiste veut faire éprouver est ce qu'il appelle « la redondance » de ces images, c'est-à-dire le fait que toutes se réfèrent à un acte répété, chaque fois insupportable et injustifiable, la destruction d'un corps humain. Si leur « provenance incertaine » contribue à faire éprouver cette redondance, le geste qui glisse sur l'écran impose une promiscuité insupportable avec la violence par le rapport qu'il établit entre le toucher et le voir. 
L'action de cette main peut en évoquer lointainement une autre, celle de Saint Thomas. Si, dans le cas de l'apôtre, le toucher prend le relais de la vue pour obtenir une preuve tangible, indubitable de la plaie, l'œuvre de Hirschhorn fait se rencontrer des images de corps détruits et un geste de caresse sans affect, geste qui, comme le dit l'artiste, est « froid », « glisse très vite » : il est « terrible », il est « non engagé » (Hirschhorn « Insoutenables destructions de corps »). Par sa régularité et son caractère répétitif, l'effleurement de la surface tactile semble participer d'un désir de voir, à la fois intarissable et indifférent. Si, comme l'écrit W.J.T Mitchell, on peut assimiler la circulation des images sur internet à « une maladie infectieuse, dont les formes de vie invasives croissent et mutent plus vite que nos défenses ne peuvent les combattre » (121), le geste filmé par Hirschhorn assimile cette « maladie » à un état d'insensibilité, d'anesthésie. Ce qui rend cette œuvre insoutenable, c'est autant les corps détruits qu'elle exhibe que ce que ce geste exprime ; c'est l'abîme qui s'y manifeste entre deux réalités corporelles, celle des victimes et celle d'un « sujet digital».

\section{Des gestes, des corps}

Si l'œuvre de Hirschhorn définit ce sujet comme un corps qui, en un geste ambigu, " touche la réalité », What Shall We Do Next? $\neq 2$ semble se tenir loin de ce réel. Les danseurs se livrent à ce qui parait être des activités domestiques, bureautiques, ludiques, comme désintéressés par les effets que leurs manipulations sont censées produire. Le réel semble ici se résumer à ce monde clos, à cet espace autarcique de fonctionnement fluide des appareils. Cependant la chorégraphie comporte des gestes et des poses qui excèdent cet univers policé et virtuel. Dans une brève séquence, des danseurs miment la pratique de jeux vidéo et évoquent, ce faisant, la place que ceux-ci ont prise dans la réalité des entraînements militaires (Citton Gestes d'humanités 73). Mais c'est surtout les effets produits par les machines sur les corps, sur leur réalité physique, que l'œuvre fait percevoir. Ainsi, l'une des danseuses raconte les conditions d'une expérience durant laquelle on demande à un sujet, dont la plante des pieds a été enduite d'oxyde de fer, de suivre une ligne tracée au milieu d'une longue feuille de papier clouée au sol. Ce récit se réfère à la méthode employée dans les années 1880 par Georges Gilles de la Tourette pour son étude de la motricité humaine. Faire référence à ces recherches permet à Prévieux d'évoquer la rationalisation dont le mouvement du corps humain est a été l'objet depuis le XIX ème siècle ainsi que la pathologie à laquelle Georges Gilles de la Tourette a donné son nom ; celle-ci se manifeste par des tics, des mouvements incontrôlés, par « une catastrophe généralisée de la sphère gestuelle » (Agamben 33). Pour Giorgio Agamben, les études de Georges Gilles de la Tourette, s'inscrivent dans « une époque [qui] a perdu ses gestes » (33). Si la formule est l'expression métaphorique des bouleversements que connaît la société de cette époque, elle désigne aussi, plus littéralement, l'impact de l'industrialisation 
sur les corps et leur motricité. Les différentes formes d'attention pour les gestes ${ }^{9}$ qui s'expriment alors, vont de pair avec cette « perte ». What Shall We Do Next? $\neq 2$ cherche manifestement à mettre en rapport ce passé « technico-corporel » avec une gestuelle d'aujourd'hui et de l'avenir. Outre les expériences de Georges Gilles de la Tourette, l'œuvre évoque, via la voix-off, le trouble physique appelé « bras de verre » dont les télégraphes souffraient « à force de taper sur un manipulateur morse mal placé ${ }^{10}$. Après que la voix off a évoqué cette pathologie, certains danseurs se placent à proximité d'une surface colorée murale. La caméra cadre les mouvements que l'une de leurs mains réalise sur fond bleu et vert. Cette suite de plans est une référence explicite au film d'Yvonne Rainer, Hand Movie de $1966^{11}$. Mais alors que celle-ci montre la diversité chorégraphique que la main peut produire, seule, les danseurs de What Shall We Do Next? $\neq 2$ répètent frénétiquement quelques mouvements contraints, crispés. Le film donne la sensation que la main est devenue un organe difforme ${ }^{12}$ agité de soubresauts ou de tics, comme si l'usage des appareils avait produit des mutations physiques.

Certains passages de l'œuvre font naître ce type d'étrangetés par la réalisation de mouvements qui ne constituent pas véritablement des gestes, du fait de leur très faible amplitude ou de leur caractère interne ; par exemple, une danseuse s'applique à faire buter sa langue sur la paroi de sa joue droite, mouvement qu'un gros plan sur le visage rend visible. Cette action s'engage alors que la voix off parle de différents instruments de captation dont un, capable de « détecter les mouvements de la langue grâce à un microphone placé dans son oreille ${ }^{13}$. Pendant un long plan silencieux, la danseuse, le regard fixe, la bouche close, se livre à une étrange gymnastique dont on perçoit le déroulement par les gonflements intermittents de sa joue. Prévieux a sans doute pensé à l'œuvre de Marcel Duchamp, With my Tongue in my Cheek (1959), cet autoportrait où l'artiste s'est représenté de profil avec une joue gonflée, figurée par un bas-relief de plâtre. À propos du titre et de l'attitude que Duchamp a choisis, Rosalind Krauss fait le constat suivant : "Avoir la "langue dans la joue", c'est être ironique ${ }^{14}$ mais c'est aussi évidemment perdre le pouvoir de la parole, c'est-à-dire abandonner le langage et être abandonné par lui » (87).

Agamben souligne que les études de Georges Gilles de la Tourette sont contemporaines de celles de Muybridge en photographie et considère que le cinéma des premiers temps n'est pas tant un art du mouvement que du geste (34).

10 Traduction par l'auteure des propos de la voix off dans What Shall We Do Next?.

11 Hand Movie d'Yvonne Rainer (1966) est un film 8 mm, noir et blanc, muet (8'). URL : https://www.youtube.com/ watch? $=5 x$ SKgl4Dd5o (page consultée le 14 novembre 2018).

12 Julien Prévieux dit avoir voulu produire un équivalent des dessins de mains biscornues que comportent certains des brevets.

13 Traduction par l'auteure des propos de la voix off dans What Shall We Do Next?

14 «With my tongue in my cheek » est l'équivalent de « mon œil ! » en français. 
Yves Citton considère que « la plupart de nos gestes sont à la fois machinés, en ce qu'ils s'inscrivent dans des séries d'opérations programmées à l'avance, et machinants, en ce qu'ils utilisent les programmes en question pour intervenir de façon intentionnelle sur le déroulement des événements » (Gestes d'humanités 65). What Shall We Do Next? et Touching Reality montrent cette « ambivalence entre aliénation et maîtrise » (66) en la situant sur le terrain des technologies numériques. Les représentations que les artistes en donnent paraissent sans commune mesure : au monde clinique et ouaté mis en place par Prévieux s'oppose la violence réelle que Hirschhorn prend le parti d'exposer. Mais ces œuvres présentent toutefois des points de concordance : ils résident dans la douceur dérangeante, dans l'impassibilité glaçante qu'elles attribuent à ces gestes de maîtrise et dans le mutisme dont les sujets agissants, connectés, semblent être frappés.

\section{OUVRages Cités}

AGAMBEN, Giorgio. « Notes sur le geste ». Traffic 1 (hiver 1991) : 33-34. URL : http://lemagazine.jeudepaume.org/2013/04/giorgio-agamben-notes-sur-le-geste/ (page consultée le 14 novembre 2018).

CITTON, Yves. Gestes d'humanités. Anthropologie sauvage de nos expériences esthétiques. Paris : Armand Colin (coll. Le Temps des idées), 2012.

—. Renverser l'insoutenable. Paris : Seuil, 2012.

DUCHAMP, Marcel, With my Tongue in my Cheek, 1959. Plâtre, crayon et papier monté sur bois. 25 x $15 \mathrm{~cm}$. Collection particulière.

HIRSCHHORN, Thomas. «Insoutenables destructions de corps ». Entretien avec Hugo Vitrani (2012). URL : http://www.dailymotion.com/video/xshfl0 (page consultée le 14 novembre 2018).

—. «Pourquoi est-il important - aujourd'hui - de montrer et regarder des images de corps humains détruits ?». Journal de la Triennale $\neq 2$ (Paris : Palais de Tokyo, 2012) : 62-67. URL : http://www.cnap.fr/sites/default/ files/article/123910_le-journal-de-la-triennale--2--4tetesetuneoreille--emilierenard.pdf (page consultée le 14 novembre 2018).

—. Touching Reality, 2012. Vidéo (4'45'). URL : https://vimeo.com/55482318 (page consultée le 14 novembre 2018).

KRAUSS, Rosalind. « Marcel Duchamp ou le champ imaginaire». Le Photographique. Pour une théorie des écarts. Trad. de l'anglais par Marc Bloch et Jean Kempf. Paris : Macula, 1990. 71-88.

MITCHELL, W.J.T. Cloning Terror ou la guerre des images du 11 septembre au présent. Trad. de l'anglais par Maxime Boidy et Stéphane Roth. Paris : Les Prairies ordinaires (coll. «Penser/croiser »), 2011. 
PRÉVIEUX, Julien. Entretien avec Olivier Poncet (12 mars 2015), dans le cadre de la journée d'études « Le geste a la parole. Interactivité et tangibilité ». HEAR (9 avril 2015). URL : https://vimeo.com/124109993 (page consultée le 14 novembre 2018).

—. What Shall We Do Next? $\neq 1$, en cours depuis 2006. Animation 3D (3'54'’). URL : https://vimeo.com/59793317 (page consultée le 14 novembre 2018).

—. What Shall We Do Next? $\neq 2,2014$. Vidéo (16'47'”). URL : https://vimeo.com/111013619 (page consultée le 14 novembre 2018).

RAINER, Yvonne. Hand Movie, 1966. Film 8 mm, noir et blanc, muet (8'). URL : https://www.youtube.com/ watch? $\mathrm{v}=5 \mathrm{xSKg}$ 14Dd5o (page consultée le 14 novembre 2018).

ROPARS, Fabian. «Faut-il dire numérique ou digital ? ». Blog du Modérateur (11 février 2015). URL : https:// www.blogdumoderateur.com/numerique-ou-digital/ (page consultée le 14 novembre 2018).

SIMONDON, Gilbert. Du mode d'existence des objets techniques. 1958. Paris : Aubier, 1989. 